\title{
Búsqueda de un tratamiento farmacológico para la dependencia de la marihuana
}

\author{
José Antonio Ramos Atance \\ Departamento de Bioquímica y Biología Molecular. Facultad de Medicina. Universidad Complutense.. \\ Enviar correspondencia a: \\ José A. Ramos Atance. Dpto. de Bioquímica. Facultad de Medicina. Universidad Complutense. Ciudad Universitaria. \\ 28040 Madrid. e-mail: jara@med.ucm.es
}

\section{RESUMEN}

No se ha encontrado todavia ningún tipo de fármaco que responda adecuadamente a las demandas de tratamiento realizadas por un número creciente de consumidores de marihuana. Por un lado, se han ensayado compuestos que están siendo utilizados para aliviar los síntomas asociados a la abstinencia al consumo de otras drogas de abuso, sin que los resultados hayan sido positivos en este caso. Entre los agonistas y antagonistas del receptor de cannabinoides, solo el uso controlado de $\delta$-9-tetrahidrocannabinol (THC), ha producido resultados interesantes para el tratamiento de la abstinencia. Pero presenta la limitación de que no disminuye el deseo de consumo. Una nueva línea de investigación esta relacionada con la funcionalidad del sistema endocannabinoide, y se basa en el diseño de fármacos que aumenten la vida media de la anandamida en el cerebro de las personas en tratamiento.

Palabras clave: marihuana, $\delta$-9-tetrahidrocannabinol, rimonabant, inhibidores de recaptación, sistema endocannabinoide, anandamida.

\section{ABSTRACT}

No kind of pharmaceutical has been found that responds adequately to the demands for treatment being made by an increasing number of marijuana users. There have, however, been trials of compounds currently being used to alleviate the symptoms associated with abstinence from other drugs, but the results have not been positive in this case. Among compounds acting as agonists or antagonists of cannabinoid receptors, only the controlled use of $\delta$-9-tetrahydrocannabinol (THC) has produced interesting results in treating abstinence. But, there are limitations on the use of $\mathrm{THC}$, as it does not reduce the craving. There is a new line of research relating to the function of the endocannabinoid system, based on the design of compounds that increase the half-life of anandamide in the brain of patients undergoing treatment.

Key words: marijuana, $\delta$-9-tetrahydrocannabinol, rimonabant, uptake-inhibitors, endocannabinoid systems, anandamide.

\section{¿DISPONEMOS DE FÁRMACOS PARA EL TRATAMIENTO DE LA DEPENDENCIA DE MARIHUANA?}

D e todos es sabido como la utilización de la metadona, a finales de los sesenta, generalizo un tipo de tratamiento para la dependencia opiácea que ha sido ampliamente utilizado en la práctica médica. En unos casos el "mantenimiento con metadona" ha conseguido que la retirada del consumo de la droga haya sido menos "costosa" para el paciente y en otros casos ha permitido su permanencia en unas condiciones de control sanitario de las que no disponía durante el periodo de consumo de los opiáceos ilegales.
Este tratamiento no fue satisfactorio en todos los casos lo que llevo a la búsqueda de otros fármacos, entre los que se encuentra la propia heroína, que fue utilizada en el pasado y esta volviendo a serlo actualmente en algunos ensayos clínicos. Se han ensayado otros agonistas opiáceos, como el levo-alfa acetilmetadol (LAAM), antagonistas como la naltrexona y agonistas parciales como la buprenorfina. Por otro lado, ha quedado bastante claro que el abordaje farmacológico necesita ser complementado con otras medidas como el tratamiento psicoterapéutico individual o en grupo y la intervención social (Leza, 2003).

En el caso de la dependencia a marihuana nos encontramos con la ausencia de la farmacología adecuada para el tratamiento de aquellas personas que desean abandonar su consumo. Los argumentos para 
justificar este hecho pueden ser muy variados y van desde la ampliamente extendida creencia de que el abuso de esta droga no presenta la problemática que suele aparecer asociada al consumo de otras drogas hasta que ni tan siquiera produce un verdadero síndrome de dependencia. Incluso existe la idea que no es una droga sino mas bien un potente recurso terapéutico. Estas creencias pueden estar asociadas a que los efectos negativos de la marihuana no suelen ser tan dramáticos como los descritos para la heroína y a que los síntomas asociados a la retirada de su consumo son más suaves, llegando en ocasiones a no ser percibidos por el propio consumidor.

Que la marihuana pueda crear dependencia en sus consumidores ha sido objeto de controversia durante mucho tiempo, pero cada vez es mayor el número de personas que solicitan tratamiento al comprobar que son incapaces de abandonar su consumo. El retraso en la aparición de los síntomas, su dispersión en el tiempo y la dificultad de su identificación pueden estar relacionados con la larga vida media del $\delta$-9-tetrahidrocannabinol (THC), que es su principal componente psicoactivo, lo que contribuye a las dudas acerca de la relevancia clínica del síndrome de abstinencia.

Sin embargo, investigaciones recientes demuestran que el consumo elevado de esta sustancia puede producir, tras su abandono, la aparición de síntomas de abstinencia, entre los que se encuentran irritabilidad, dificultades para dormir, disminución del apetito, perdida de peso, trastornos intestinales, ansiedad, irritabilidad, comportamiento agresivo, depresión, nerviosismo y deseo de volver a consumir. La vuelta al consumo puede representar el intento de aliviar estos síntomas, que suelen aparecer al cabo de uno o dos días de abstinencia y alcanzan su máxima intensidad entre dos y seis días después del abandono del consumo. El desarrollo de los síntomas suele ser gradual y su duración es bastante prolongada pudiendo llegar a durar hasta catorce días (Budney et al. 2003).

\section{¿CÓMO ACTÚA LA MARIHUANA SOBRE EL CEREBRO?}

Es conocido que las drogas de abuso ejercen sus efectos negativos sobre el cerebro mediante procesos neuroadaptativos en los que aparece implicado el denominado "sistema de recompensa cerebral". Este sistema es activado por diferentes drogas de abuso entre las que se encuentra la marihuana. Recientemente se ha demostrado la existencia en el cerebro de un sistema endocannabinoide, el cual forma parte del sistema de recompensa cerebral (Maldonado, 2002). Su principal componente endógeno es la anandamida, que actúa sobre el denominado receptor CB1, dando lugar a la correspondiente respuesta biológica.
En los estudios realizados en modelos animales se ha visto que la administración crónica de THC afecta al sistema endocannabinoide, aumentando el contenido de anandamida en el cerebro anterior limbico y disminuyendo en diversas regiones cerebrales, el número de receptores CB1 y su funcionalidad, lo que produce la desensibilización de los mecanismos celulares acoplados a la activación del receptor CB1. El aumento en la producción de anandamida podría representar una respuesta adaptativa a la continuada presencia de THC en el cerebro del animal. Su misión podría ser contrarrestar parte de los síntomas del comportamiento de búsqueda de droga (di Marzo et al. 2000).

Sin embargo, los cannabinoides no suelen reproducir en animales de laboratorio el síndrome de abstinencia espontáneo que aparece cuando se interrumpe la administración crónica de algunas drogas de abuso. Para su desarrollo se necesita la administración del antagonista del receptor CB1, SR141716 A. Su inyección precipita un síndrome de abstinencia en ratas hechas tolerantes a THC, durante el cual se revierte el aumento de la concentración de anandamida producido en la corteza anterior límbica por la administración crónica de THC. (González et al. 2004).

Algo parecido a lo descrito en animales puede estar ocurriendo en el cerebro humano tras el consumo continuado de marihuana. La alteración de la funcionalidad del sistema endocannabinoide, producida por el consumo de marihuana, puede contribuir a la "resistencia" que aparece en el individuo al abandono de su consumo. Ello conlleva la conveniencia de prestar algún tipo de ayuda a las personas que lo demanden. Sin embargo, el aumento en el número de peticiones de tratamiento para dejar de consumir, no viene acompañada del adecuado conocimiento de las estrategias a utilizar ni dispone de fármacos que hayan demostrado su utilidad para evitar el consumo de esta droga.

\section{TIPOS DE ESTRATEGIAS UTILIZADAS EN ELTRATAMIENTO DE LA DEPENDENCIA A MARIHUANA}

Todos los intentos de establecer algún tipo de tratamiento deben partir de la base de que la adicción a la marihuana conlleva procesos patológicos en los estados físicos, mental (cognitivo) y emocional (estado de ánimo) del individuo adicto. La solución al problema debe tratar de conseguir la recuperación de los "estados normales" del individuo.

La intoxicación por cannabis no suele requerir ningún tipo de abordaje, salvo que se asocie a alguna de las complicaciones, como estados de crisis 
de angustia, trastorno psicótico inducido o trastorno confusional.

En cuanto al abandono del consumo, si la implicación con la droga no es muy severa, se suelen elegir modalidades poco intrusivas como el tratamiento ambulatorio o bien la participación en grupos de autoayuda. El programa comienza con la desintoxicación ambulatoria del paciente, cosa que significa abstinencia de consumo y eventual administración de ansiolíticos.

En la posterior etapa de deshabituación se pueden utilizar diferentes tipos de estrategias psicológicas entre las que cabe destacar las cognitivo-conductuales. Se trata de aumentar la conciencia de problema y la motivación por abandonar definitivamente el consumo. Los tratamientos eficaces incluyen consejo y orientación, enseñanza de habilidades adecuadas, y refuerzo de la conducta alternativa apropiada (Solé J. 2001)

En el caso de consumo en paralelo de otras drogas, o bien si aparece sintomatología psiquiátrica, habrá que plantearse otro tipo de intervención. No debe olvidarse que los sujetos consumidores de cocaína suelen utilizar la marihuana para aliviar las sensaciones de ansiedad. Los consumidores de heroína pueden consumirla para potenciar los efectos del opiáceo y otros consumen alcohol para potenciar los efectos de la intoxicación por el hachís. En todos estos casos el tratamiento deberá tener en cuenta las diferentes estrategias a utilizar para cada una de las drogas consumidas.

Por otro lado, son frecuentes en las urgencias de los hospitales las complicaciones psiquiátricas derivadas de su utilización. Además, algunos pacientes psiquiátricos también suelen consumir este tipo de droga, de ahí la necesidad de una exhaustiva exploración psicopatológica que nos permita el diagnóstico psiquiátrico del trastorno asociado al consumo, así como la posible relación entre la psicopatología y el consumo de marihuana. De la adecuada identificación del trastorno psiquiátrico asociado dependera el tipo de tratamiento a establecer para la curación del paciente (Santo Domingo y Jiménez-Arriero,2003).

\section{BÚSQUEDA DE LOS FÁRMACOS MAS ADECUADOS PARA ELTRATAMIENTO DEL SÍNDROME DE ABSTINENCIA.}

Los intentos farmacológicos realizados para aliviar este síndrome son todavía escasos. En la práctica médica habitual se suele instaurar una pauta de sedación durante los días que dure la sintomatología, recomendandose el uso de diazepam.
Algunos ensayos de tratamiento realizados en la década de los ochenta utilizaron técnicas aversivas entre las que conviene destacar a nivel anecdótico, la desarrollada en Nigeria con nueve jóvenes fumadores de marihuana. El relato parece inspirado en el tratamiento a que se somete al protagonista de la película "La naranja mecánica". Los jóvenes recibían 3 inyecciones intramusculares diarias, durante cuatro días, de un emético inductor de náuseas, mientras veían reportajes en los que ellos mismos aparecían preparando y consumiendo la marihuana (Morakinyo, 1983).

El resultado de este tipo de técnicas agresivas, aunque prometedoras en su momento, no tuvieron continuidad, quizás debido al especial grado de motivación necesario para que un paciente desee someterse a un tratamiento de tipo aversivo.

Los primeros estudios realizados sobre los efectos del tratamiento farmacológico de la dependencia a marihuana fueron secundarios al tratamiento de la dependencia a otras drogas de abuso. Este es el caso de la disminución del consumo de marihuana observado en un grupo de alcohólicos que presentaban síntomas de depresión y que por este motivo habían sido tratados con flouxetina (Cornelius et al. 1998). En otro grupo de pacientes dependientes de opiáceos, el tratamiento con buspirona, no redujo apreciablemente su ansiedad, pero produjo una disminución significativa del consumo de marihuana (McRae et al. 2000).

El que la medicación antidepresiva o ansiolítica pudiera producir una disminución en la dependencia a marihuana hizo pensar en la posibilidad de que su aplicación en el periodo de abstinencia podría contribuir a la atenuación de los síntomas que contribuyen a la vuelta al consumo.

Se han probado los efectos de dos antidepresivos; la nefazodona (serzone)y el bupropion (zyban). La nefazodona disminuye algunos síntomas como la ansiedad y el dolor muscular (Haney et al. 2003), pero puede producir problemas hepáticos. El bupropion empeora el estado de animo de los participantes que se muestran mas irritables, infelices y deprimidos (Haney et al. 2001).

Posteriormente se realizó un estudio con el divalproex, que es un estabilizador del estado de ánimo utilizado para controlar síntomas de la abstinencia al alcohol y a las benzodiacepinas como son la ansiedad, el insomnio y las náuseas. En el caso de la marihuana este fármaco empeora el estado de animo, aumentando la ansiedad y altera la funcionalidad cognitiva durante la abstinencia a marihuana (Haney et al. 2004).

En todos estos casos la estrategia utilizada consistió en la reducción de los síntomas en un intento 
de hacer mas llevadera la retirada del consumo. La existencia en el mercado de un antagonista para el receptor CB1, el SR141716A (Rimonabant) hace pensar en la posibilidad de su utilización en el caso de la marihuana de una forma parecida a la que los antagonistas de los receptores mu lo están siendo en el caso de los opiáceos. El Rimonabant esta siendo probado en el tratamiento de la dependencia a alcohol y a tabaco y esta a punto de salir al mercado un tratamiento de la obesidad con este compuesto. Estos estudios pueden aportarnos datos sobre las dosis a utilizar y los posibles efectos secundarios que pudiera tener la administración del Rimonabant, pero no parecen estar siendo utilizados para el tratamiento de la dependencia a marihuana (McRae et al. 2003).

Un tratamiento que aparece como prometedor es la administración oral de THC. Se trataría de un tipo de terapia de sustitución parecida a la utilización de la metadona en el caso de los opiáceos, Los primeros resultados obtenidos indican una disminución significativa de síntomas como ansiedad, aflicción, escalofríos, alteraciones del sueño, el deseo y la reversión de la anorexia y la pérdida de peso asociadas a la abstinencia (Haney et al. 2004). Su utilización puede estar limitada por la aparición de efectos secundarios indeseables como son los psicotrópicos y los cardiovasculares. Otro problema puede ser que, como ha ocurrido en el caso de la metadona, se prolongue indefinidamente el tratamiento con este compuesto. Además, la ingestión oral de THC (60-80 mg/día) no afecta el reforzamiento positivo producido por el consumo de marihuana, por lo que continua la autoadministración de esta droga, lo que parece indicar que el tratamiento con THC solo sería útil en el periodo de abstinencia (Hart et al. 2002).

Una forma de obviar estas limitaciones y de ampliar el rango de síntomas de la abstinencia mejorados por el tratamiento puede ser el desarrollo de agentes farmacológicos que prolonguen la vida media de la anandamida. Diversos estudios animales han confirmado que el aumento de los niveles extracelulares de este compuesto produce efectos ansiolíticos lo que indica que la anandamida puede contribuir a la regulación de la emoción y de la ansiedad (Gaetani et al. 2003). La anandamida podría ser activada en respuesta a una situación ansiogénica, y esta activación formaría parte de un sistema de retrocontrol negativo para controlar la ansiedad.

Este tipo de estrategia ha sido utilizada en psiquiatría con los inhibidores de la recaptación de serotonina y puede conducir a un espectro de respuestas biológicas mas acorde con la funcionalidad fisiológica del sistema endocannabinoide que el producido por la administración de THC. Además, diversos estudios animales han mostrado que el bloqueo del transporte de anandamida produce una serie de efectos far- macológicos que son claramente distinguibles de los atribuidos al THC.

Entre los inhibidores de la recaptación de anandamida se encuentran el AM404 y el UCM 707. El primero es el que mas ha sido estudiado y su administración a ratas y a ratones aumenta los niveles endógenos de anandamida en tejidos cerebrales y en sangre (Fegley et al. 2004). El UCM707 es el inhibidor mas potente de los descritos hasta la fecha y posee una elevada especificidad por el recaptador (LópezRodríguez et al. 2003).

El aumento de los niveles de anandamida producido por la utilización de estos inhibidores quizás pudiera servir para eliminar el deseo de consumo. El que este compuesto no tenga ningún efecto psicotrópico es una ventaja frente a los estudios que se están realizando con THC (Haney et al. 2004). Además se trata de la modulación de un mecanismo endógeno del propio individuo, evitando los efectos secundarios que pueden aparecer con elTHC.

\section{CONCLUSIONES}

Queda mucho camino por recorrer hasta que dispongamos de los fármacos mas adecuados para el tratamiento de la dependencia a marihuana de una forma similar a la que están siendo utilizada en el caso de los opiáceos. Las sustancia que han servido para controlar los síntomas asociados al consumo de otras drogas de abuso no parecen conseguir los mismos resultados en el caso de la marihuana. La utilización de agonistas o antagonistas del receptor de cannabinoides esta por ahora limitada al uso controlado del THC. Todo ello ha conducido al desarrollo de nuevas vías de investigación relacionadas con la funcionalidad del sistema endocannabinoide. Un ejemplo de estas estrategias alternativas es el diseño de fármacos que aumente la vida media de la anandamida en el cerebro de las personas en tratamiento.

\section{REFERENCIAS}

Budney, A., Moore, B., Vandrey, R., y Hughes, J. (2003). The time course and significance of cannabis withdrawal. J. Abnorm. Psychol, 112,393-402.

Cornelius, J.R., Salloum, J.M., Haskett, R.F., Ehler, J.G., Jarrett, P.J., Thase, M.E. y Perel, J.M. (1998). Fluoxetine versus placebo for the marihuana use of depressed alcoholics. Addictive Behaviors, 24,111-114 . 
Di Marzo, V., Berrendero, F., Bisogno, T., Gonzalez, S., Cavaliere, P., Romero, J., Cebeira, M., Ramos, J.A. y Fernandez-Ruiz, J. (2000). Enhancement of anandamide formation in the limbic forebrain and reduction of endocannabinoid contents in the striatum of $\delta$-9-tetrahydrocannabinol-tolerant rats. J. Neurochem, 74, 1627-35.

Fegley, D., Kathuria, S., Mercier, R., Li, C., Makriyannis, A. y Piomelli D. (2004). Anandamide transport is independent of fatty acid amide hydrolase activity and is blocked by the hydrolisis resistant inhibitor AM1172. Proc. Natl. Acad. Sci. USA, 99,8756-61.

Gaetani, S., Cuomo, V. y Piomelli D. (2003). Anandamide hydrolisis: a new target for anti-anxiety drugs? Trends Mol. Medicine, 9,474-8.

González, S., Fernandez-Ruiz, J., di Marzo, V., Hernandez, M.L., Arevalo, C., Nicanor, C., Cascio, MG., Ambrosio, E. y Ramos, J.A. (2004). Behavioural and molecular changes elicited by acute administration of SR141716 to $\delta$-9-tetrahidrocannabinol-tolerant rats; an experimental model of cannabinoid abstinence. Drug and Alcohol Depend, 74,159-770.

Haney, M., Ward, A.S., Comer, S.D., Hart. C.L., Foltin, R.W. y Fischman, M.W. (2001). Bupropion SR worsens mood during marihuana withdrawal in humans: Psychopharmacology, 155,171-79.

Haney, M., Hart, C., Ward, A.S. y Foltin, R.W. (2003). Nefadone decreases anxiety during marihuana withdrawal in humans. Psychopharmacology, 165,15765.

Haney, M., Hart, C., Vosburg, S., Nasser, J., Bennett, A., Zubaran, C. y Foltin, R,W. (2004). Marijuana withdrawal in humans: effects of oral THC or divalproex. Neuropsychopharmacology, 29,158-70.

Hart, C.L., Haney, M., Ward, A.S., Fischman, M.W. y Foltin, M,W. (2002). Effects of oral THC maintenance on smoked marijuana self-administration. Drug Alcohol Depend, 67,301-9.

Leza, J.C. Opiáceos (III) (2003). Tratamiento de la dependencia opiácea (I). Agonistas opiáceos. En P. Lorenzo, JM Ladero, Leza JC Lizasoain I (Eds.), Drogodependencias: Farmacología. Patología. Psicología. Legislación. (pp 115-125) Editorial Medica Panamericana. Madrid.

López-Rodriguez, M.L., Viso, A., Ortega-Gutierrez, S., Fowler, Ch., Tiger, G., De Lago, E., Fernandez-Ruiz, J. y Ramos, J.A. (2003). Design, synthesis and biological evaluation of new inhibitors of the endocannabinoid uptake: comparison with effects on fatty acid amidohydrolase. J. Med. Chem, 46,1512-22.

Maldonado, R. (2002). Study of cannabinoid dependence in animals. Pharmacol Ther, 95,153-64.

McRae, A.L., Sonne, S.C. y Brady, K.T. (2000). The use of buspirone for the treatment of persistent anxiety in methadone-maintained patients. Paper presented at the College on Problems of Drug Dependence 62 Annual Scientific meeting San Juan, Puerto Rico.

McRae, A.L., Budney, A.J. y Brady KT. (2003). Treatment of marihuana dependence: a review of the literature. J. Substance Abuse Treatment, 24,369-376.

Morakinyo, O. (1983). Aversión therapy of cannabis dependence. Drug Alcohol Depend, 12,287-293.

SantoDomingo, J. y Jimenez Arriero M.A. (Coordinadores). (2003). Capitulo IV Cannabis, en Consenso de la Sociedad Española de Psiquiatría sobre el diagnóstico y tratamiento del alcoholismo y otras adicciones. (Pp. 85-91). Sociedad Española de Psiquiatria.

Solé, J. (2001). Consideraciones Terapéuticas, en Cannabinoides: Aspectos Psiquiátricos y Bioquímicos. Solé, J. y Ramos, J.A, (Eds.). (pp.75-92). Barcelona, Ediciones Rol S.A. 
